07

\title{
О полях напряжений в гетерогенных средах, определяемых методом акустической эмиссии
}

\author{
() В.Л. Гиляров, Е.Е. Дамаскинская
}

Физико-технический институт им. А.Ф. Иофрфе РАН, Санкт-Петербург, Россия

E-mail: Vladimir.Hilarov@mail.ioffe.ru

Поступила в Редакцию 7 декабря 2020 г.

В окончательной редакции 7 декабря 2020 г.

Принята к публикации 7 декабря 2020 г.

\begin{abstract}
Предложен метод расчета локальных внутренних напряжений на основе анализа пауз между последовательными сигналами акустической эмиссии в материалах при их механическом нагружении. Метод основан на кинетической концепции прочности С.Н. Журкова. Изучена эволюция этих напряжений в процессе разрушения двух различных по структуре материалов.
\end{abstract}

Ключевые слова: механические напряжения, кинетическая концепция прочности, акустическая эмиссия.

DOI: 10.21883/FTT.2021.04.50718.256

\section{1. Введение}

Информация о полях напряжений, действующих в материалах в процессе их деформирования или разрушения, представляется чрезвычайно важной как для развития представлений о самих этих процессах, так и для безопасной эксплуатации материалов и конструкций из них изготовленных. Используя метод акустической эмиссии можно получить такие сведения на основе анализа времен ожидания последовательных событий акустической эмиссии (AЕ) (пауз между событиями).

Согласно кинетической концепции Журкова [1] процесс разрушения представляет собой последовательность элементарных актов разрушения (термических флуктуаций). Среднее время ожидания флуктуации определяется формулой Журкова

$$
\tau=\tau_{0} \cdot \exp \left(\frac{U_{0}-\gamma \cdot \sigma}{k \cdot T}\right),
$$

где $U_{0}$ - энергия активации процесса, $\tau_{0}$ - характерное время тепловых колебаний атомов (обе эти величины предполагаются константами материала), $\sigma$ - внешнее приложенное напряжение, $k$ - постоянная Больцмана, $T$ - абсолютная температура. Величина $\gamma$ носит название активационного объема и имеет различные трактовки. Наиболее часто ее считают равной произведению атомного объема на коэффициент локальных перенапряжений [2].

В настоящей работе мы будем использовать несколько иную трактовку процесса разрушения. Предположим, что материал (образец, конструкция) находится в состоянии равновесия в отсутствии внешних напряжений. Внешняя механическая нагрузка выводит материал из равновесного состояния. Система пытается перейти в новое равновесие, проходя через череду метастабильных состояний. В каждом таком состоянии система живет время $\Delta t_{i}$, которое представляет собой паузу между последовательными акустическими событиями и известно из эксперимента. Акустическое событие (сигнал) возникает в результате перестройки структуры материала. Это может быть двойникование, образование или прорастание трещины и т.д. [3-5]. Переход в следующее метастабильное состояние происходит термофлуктуационно по закону Журкова вида (1), однако, это происходит локально в конкретном месте образца в определенный момент времени. Эту локальность учтем зависимостью действующего напряжения от координат и времени $\sigma=\sigma(\mathbf{r}, t)$. При этом константу $\gamma$ будем считать также константой материала. В общем случае в месте разрыва существует сложное напряженное состояние, в котором присутствуют несколько компонент тензора напряжений. Поскольку величина $\gamma$ в (1) скалярна, величина $\sigma$ тоже должна быть скаляром. Простейший скаляр размерности напряжения, который можно составить из компонент тензора напряжений, представляет собой первый инвариант этого тензора. Таким образом, будем предполагать, что в (1) фигурирует именно он. Учитывая эти факты, формулу (1) можно переписать в виде

$$
\sigma\left(\mathbf{r}, t_{i}\right)=\frac{U_{0}+k \cdot T}{\gamma} \cdot \ln \left(\frac{\tau_{0}}{\Delta t_{i}}\right) .
$$

Таким образом, локальное напряжение можно определить, зная время жизни $i$-того метастабильного состояния (паузу между акустоэмисионными сигналами) и температуру (в общем случае локальную). При выделении упругой энергии в результате локального разрушения возможно повышение температуры в окрестности, однако стоит отметить, что это происходит уже после элементарного аррениусовского акта. Поэтому температуру в (2) можно условно считать постоянной.

Заметим, что напряжение зависит от акустоэмиссионной паузы логарифмически. Поэтому точности из- 
Таблица 1. Минеральный состав гранита Westerly

\begin{tabular}{c|c|c|c|c|c}
\hline Минерал & $\begin{array}{c}\text { Quartz } \\
\text { (кварц })\end{array}$ & $\begin{array}{c}\text { Plagioglase } \\
\text { (плагиоклаз) }\end{array}$ & $\begin{array}{c}\text { K-feldspar } \\
\text { (полевой шпат) }\end{array}$ & $\begin{array}{c}\text { Вiotite } \\
\text { (биотит) }\end{array}$ & $\begin{array}{c}\text { Muscovite } \\
\text { (мусковит) }\end{array}$ \\
\hline & $28 \%$ & $33 \%$ & $33 \%$ & $3.5 \%$ & $1.9 \%$ \\
\hline Плотность & $2600-2650 \mathrm{~kg} / \mathrm{m}^{3}$ & $2620-2760 \mathrm{~kg} / \mathrm{m}^{3}$ & $2560 \mathrm{~kg} / \mathrm{m}^{3}$ & $2800-3400 \mathrm{~kg} / \mathrm{m}^{3}$ & $2760-3100 \mathrm{~kg} / \mathrm{m}^{3}$
\end{tabular}

Таблица 2. Минеральный состав песчаника Berea

\begin{tabular}{c|c|c|c|c|c}
\hline Минерал & $\begin{array}{c}\text { Quartz } \\
\text { (кварц) }\end{array}$ & $\begin{array}{c}\text { Plagioglase } \\
\text { (плагиоклаз) }\end{array}$ & $\begin{array}{c}\text { K-feldspar } \\
\text { (полевой шпат) }\end{array}$ & $\begin{array}{c}\text { Каolinite (каолинит) } \\
\text { (силикат алюминия) }\end{array}$ & $\begin{array}{c}\text { Сalcite } \\
\text { (кальцит) СаСО } 3\end{array}$ \\
\hline & $85-90 \%$ & $1-2 \%$ & $3-6 \%$ & $2-6 \%$ & $6-8 \%$ \\
\hline Плотность & $2600-2650 \mathrm{~kg} / \mathrm{m}^{3}$ & $2620-2760 \mathrm{~kg} / \mathrm{m}^{3}$ & $2560 \mathrm{~kg} / \mathrm{m}^{3}$ & $2600 \mathrm{~kg} / \mathrm{m}^{3}$ & $2710 \mathrm{~kg} / \mathrm{m}^{3}$
\end{tabular}

мерения паузы во многих случаях недостаточно для сколь-нибудь верного расчета величины напряжений в (2). Однако мы будем интересоваться не самим значением величины напряжения, а характером изменений напряжений в процессе разрушения материала.

\section{2. Описание эксперимента}

В работе исследовалось накопление дефектов в природных материалах: гранит Westerly [6] - существенно гетерогенный материал с практически нулевой пористостью, в котором средний размер зерна составляет $75 \mu \mathrm{m}$ [7]; песчаник Berea - хрупкая осадочная порода с характерным размером зерна $20 \mu \mathrm{m}$, пористость порядка 15-20\% [8]. Минеральный состав материалов приведен в таблицах 1 и 2.

Образцы (цилиндры $d=10 \mathrm{~mm}, h=20 \mathrm{~mm}$ ) были подвергнуты одноосному квазистатическому сжатию со скоростью нагружения (смещения нагружающих плит) $5 \mu \mathrm{m} / \mathrm{min}$. Механическое напряжение прикладывали параллельно оси цилиндра. Сжатие осуществлялось до усилия, равного 0.9 от $F_{\max }\left(F_{\max }-\right.$ разрушающая нагрузка, определенная в предварительных экспериментах). Затем образец выдерживался при постоянной деформации до тех пор, пока активность АE не спадала до нуля.

В результате, удалось с одной стороны, сформировать магистральную трещину, а с другой - сохранить целостность образца.

На рис. 1 представлены характерные временные зависимости деформации и активности $\mathrm{AE}$ (число сигналов в единицу времени). Видно, что активность акустической эмиссии значительно нарастает при подходе к максимальной деформации, а затем (при удержании постоянной деформации) спадает со временем до нуля.

До механических испытаний выполнялась рентгеновская компьютерная томография образцов (с простран-

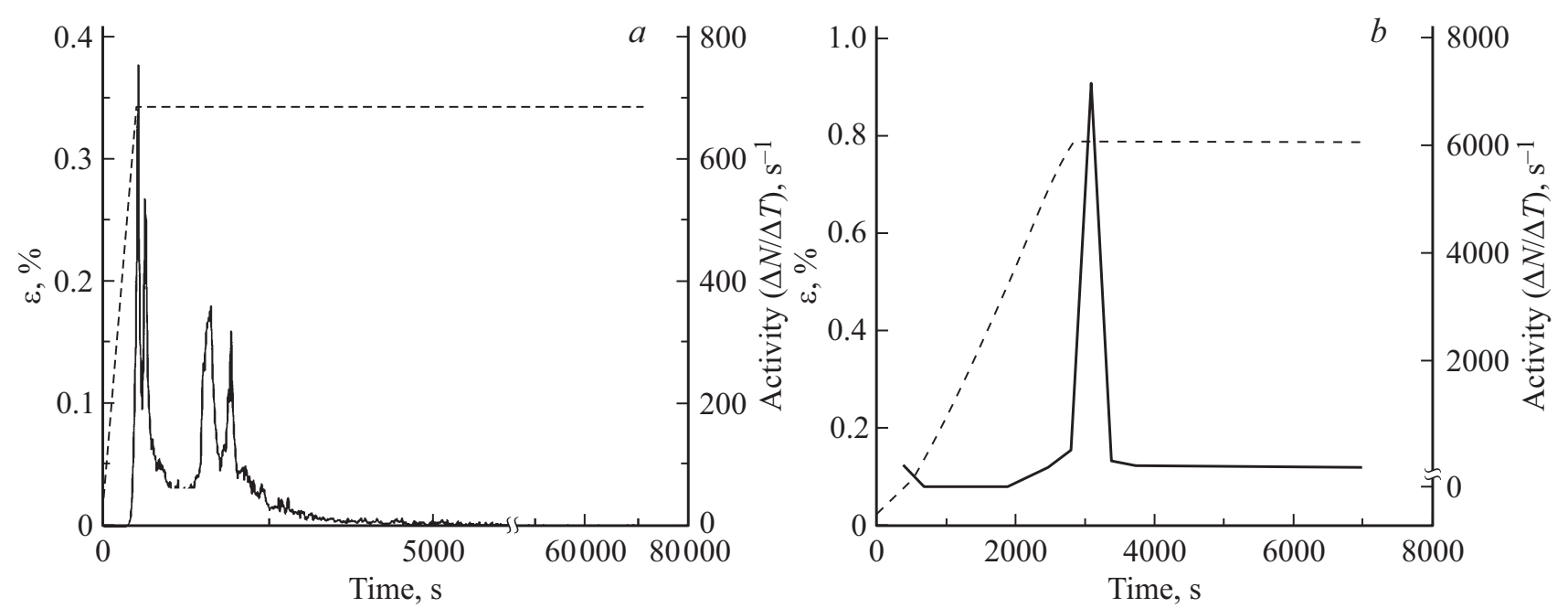

Рис. 1. Изменение деформации (штриховая линия), активности акустической эмиссии (сплошная линяя) в процессе эксперимента: $a$ - песчаник Berea, $b-$ гранит Westerly. 

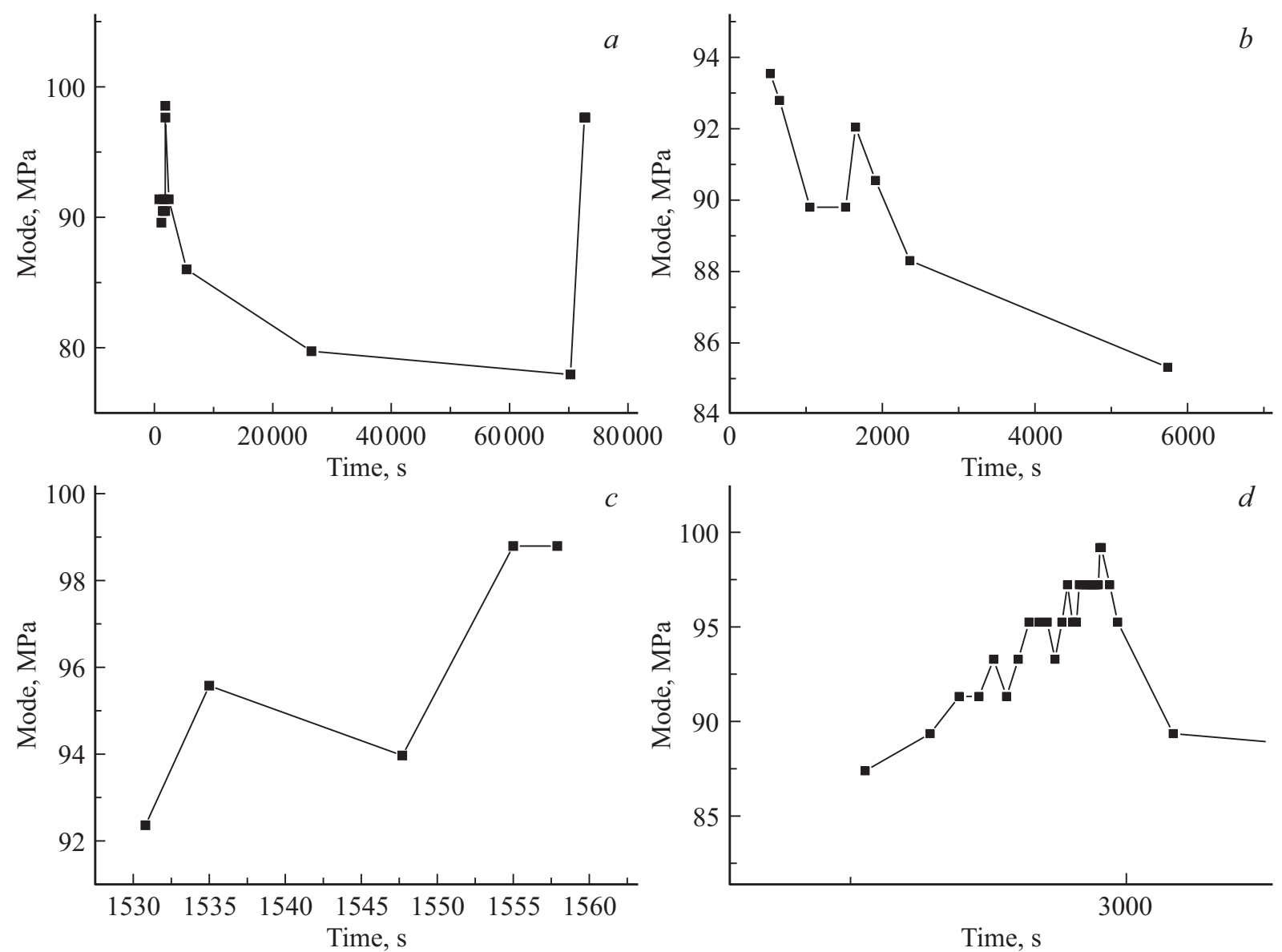

Pис. 2. Зависимости от времени модального значения напряжения для образцов из песчаника Berea $(a, b)$ и гранита Westerly $(c, d)$.

ственным разрешением $3 \mu \mathrm{m})$. Отобраны образцы без структурных аномалий, которые могут выступать концентраторами напряжений и источниками накопления повреждений.

В процессе нагружения образца производилась регистрация сигналов акустической эмиссии в реальном времени. Два пьезопреобразователя крепились к торцам образца, что дало возможность решать задачу линейной локации источников сигналов АЕ. Каждый сигнал акустической эмиссии характеризовался временем излучения, координатой источника по высоте образца и энергией. Точность определения координат источника сигнала АЕ составляла около $2 \mathrm{~mm}$. Детали эксперимента были описаны ранее, например, [9].

Следует отметить, что в любом эксперименте регистрируются не все сигналы акустической эмиссии, а только их часть (это связано с возможностями аппаратуры). В таком случае применение формулы (2) напрямую невозможно, поскольку зарегистрированная пауза $\Delta t_{i}$ может состоять из нескольких меньших, содержащих в себе пропущенные сигналы

$$
\Delta t_{i}=\sum_{j} \Delta t_{i j}=\tau_{0} \cdot \exp \left(\frac{U_{0}}{k \cdot T}\right) \cdot \sum_{j} \exp \left(-\frac{\gamma \cdot \sigma_{i j}}{k \cdot T}\right) .
$$

Каждая из этих меньших пауз описывается формулой вида (1), однако сумма экспонент в общем случае экспонентой не является. Но излучение сигналов акустической эмиссии в нашем случае происходит при значительных приложенных к образцам напряжениях, и соответственно, высоких внутренних напряжениях, для которых $\gamma \cdot \sigma_{i j} / k \cdot T \gg 1$. Тогда в сумме (3) лидирует только одна экспонента, в которой напряжение минимально, поскольку остальные спадают еще быстрее. Поэтому в этом случае сумму (3) можно заменить одной экспонентой. Полученная рассматриваемым методом оценка напряжений является оценкой снизу. К этому же приводит и конечная точность определения времен акустоэмиссионных событий.

\section{3. Основные результаты}

Расчеты проводились для четырех различных образцов, два из которых были изготовлены из гранита Westerly, а два других из песчаника Berea. Следует отметить, что энергии акустоэмиссионных событий не учитываются в настоящем расчете. Действительно, в формулах (1) и (2) фигурирует энергетический барьер, в то время как выделившаяся энергия определяется разно- 

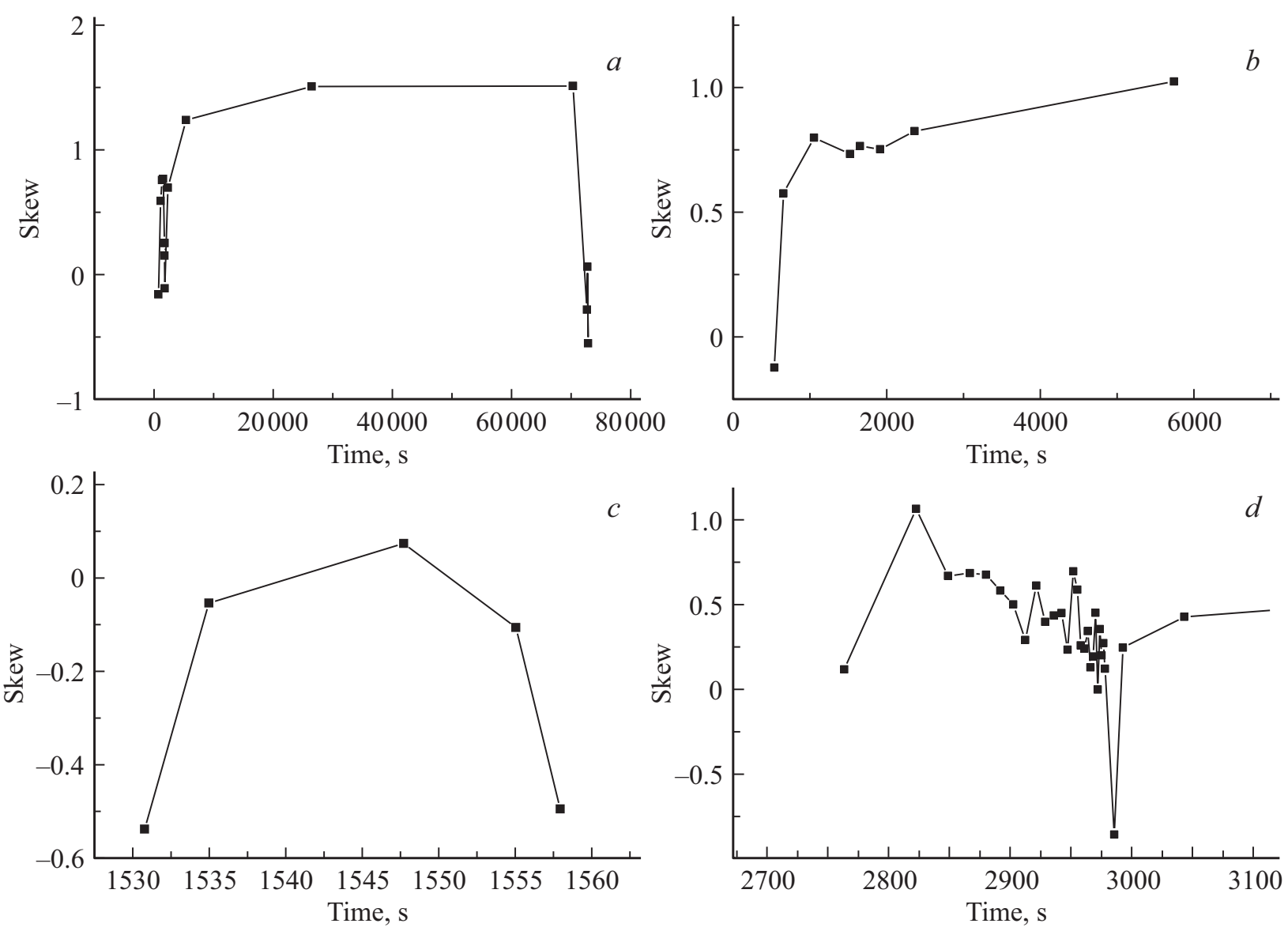

Рис. 3. Зависимости от времени коэффициента ассиметрии гистограммы напряжений для образцов из песчаника Вегеа $(a, b)$ и гранита Westerly $(c, d)$.

стью энергий начального (до элементарного акта) и конечного (после него) состояний и не связана с энергией барьера. Временные ряды данных акустической эмиссии разбивались на непересекающиеся последовательности, состоящие из 2048 или 256 событий (в том случае, когда число сигналов было невелико). Для каждой последовательности рассчитывалась гистограмма распределения величины $\sigma$, и ее статистические параметры, такие как модальные и средние значения, стандартные отклонения и коэффициенты асимметрии.

На рис. 2 приведены зависимости от времени моды гистограммы распределения для четырех различных образцов, два из которых изготовлены из песчаника Berea $(a$ и $b)$ и два из гранита Westerly ( $c$ и $d)$. Характерной особенностью этих графиков является существенное увеличение характерного значения (моды) напряжения (концентрация напряжений) в моменты максимальной акустической активности. Это неудивительно, поскольку в настоящей работе напряжения как раз и определяются по этой активности. Нужно отметить, что в силу конечной точности определения $\Delta t_{i}\left(\sim 10^{-5} \mathrm{~s}\right)$, логарифмической зависимости напряжения от $\Delta t_{i}$ и наличия незарегистрированних сигналов очень большие концентраторы напряжений не наблюдаются. Видно су- щественное отличие поведения образцов из гранита от образцов из песчаника. В периоды затишья (незначительной акустической активности) характерные напряжения в образцах песчаника в среднем уменьшаются, что говорит о релаксации напряжений в этих материалах, поскольку система находится при заданной сжимающей деформации. Напротив, в образцах из гранита Westerly напряжения в среднем увеличиваются во времени (накапливаются). Для расчета напряжений были использованы следующие значения констант: $U_{0}=160 \mathrm{~kJ} / \mathrm{mol}$; $\gamma=1 \mathrm{~kJ} / \mathrm{mol} \cdot \mathrm{MPa} ; \tau=10^{-13} \mathrm{~s}^{-1}[10]$.

На рис. 3 приведены зависимости от времени коэффициентов асимметрии (КА) для образцов песчаника Berea ( $a$ и $b)$ и гранита Westerly $(c$ и $d)$. Коэффициент асимметрии рассчитывается по формуле Skew $=E(x-\bar{x})^{3} / s t d^{3}$; т. е. равен отношению третьего центрального момента распределения величины $x$ к кубу стандартного отклонения std. Если КА равен нулю, то распределение симметрично относительно своего среднего значения $\bar{x}$ (например, нормальное распределение). При Skew < 0 у распределения растянут левый хвост, а Skew > 0 при правый.

Как следует из рис. 3, в периоды затишья, когда происходит релаксация напряжений в песчанике, коэффициент 

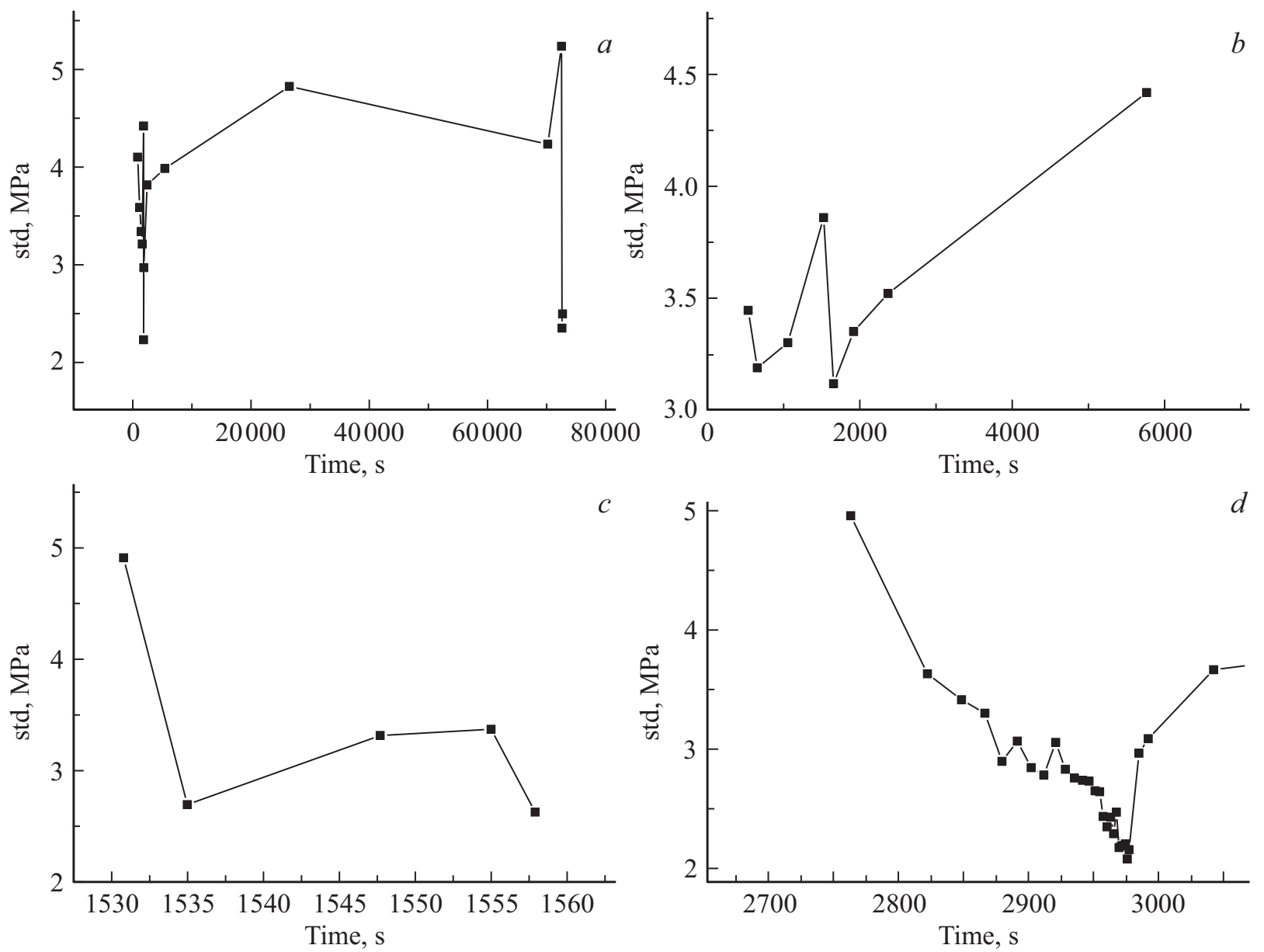

Рис. 4. Зависимости от времени стандартного отклонения гистограммы напряжений для образцов из песчаника Вегеа $(a, b)$ и гранита Westerly $(c, d)$.

асимметрии распределения напряжений увеличивается до величины $\sim 1$, т.е. правый хвост распределения тяжелее левого. Однако в периоды всплеска активности КА находится около нуля. В образцах гранита, наоборот, КА либо остается низким практически все время, либо уменьшается во времени.

Мерой ширины распределения может служить среднеквадратичное отклонение $(s t d)$. Его зависимость от времени для рассматриваемых материалов приведена на рис. 4. Как следует из этих графиков, для образцов песчаника ширина распределения во времени в среднем увеличивается, а для гранитов уменьшается.

\section{4. Обсуждение результатов}

Полученные результаты можно объяснить различным характером релаксации напряжений в песчанике и граните. Песчаник обладает значительной пористостью (содержит до $20 \%$ свободного объема). Приложение сжимающего напряжения может приводить к закрытию существующих в материале пор. Поэтому здесь возможно появление в процессе разрушения больших локальных напряжений, проявляющихся в увеличении ширины гистограммы распределения и значительном положительном коэффициенте асимметрии этого распределения, поскольку эти напряжения имеют канал релаксации. Напротив, в граните, не обладающем пористостью, напряжения во времени накапливаются. Поскольку релаксации напряжений в этом случае не происходит, это приводит к разрушению материала. Гистограмма распределения напряжений сужается, коэффициент асимметрии уменьшается или принимает отрицательные значения (что говорит об отсутствии напряжений выше некоего критического значения). Отметим, что перед разрушением для образца песчаника Berea на рис. 4, $a$ также наблюдается сужение гистограммы распределения напряжений и уменьшение КА, что говорит об исчерпании возможности релаксации напряжений перед разрушением материала. Для образца на рис. 4, $b$ этого не происходит, поскольку здесь эксперимент был остановлен задолго до момента разрушения.

Заметим, что для гранита и песчаника использовался один и тот же набор констант материала $U_{0}, \tau_{0}$ и $\gamma$, поэтому сравнить абсолютные значения рассчитанных напряжений бессмысленно. 


\section{5. Заключение}

Таким образом, предложенный, исходя из термофлуктуационной природы прочности, метод определения внутренних напряжений, представляется перспективным для выявления механизмов эволюции этих напряжений в материалах в процессе их разрушения.

\section{Финансирование работы}

Работа выполнена при финансовой поддержке Российского фонда фундаментальных исследований (грант № 19-05-00248).

\section{Конфликт интересов}

Авторы заявляют об отсутствии конфликта интересов.

\section{Список литературы}

[1] S.N. Zhurkov. J. Fracture Mechanics 1, 311, (1965).

[2] В.Р. Регель, А.И. Слуцкер, Э.Е. Томашевский. Кинетическая природа прочности твердых тел. М., Наука, (1974), $560 \mathrm{c}$.

[3] В.И. Веттегрень, В.С. Куксенко, И.П. Щербаков. ЖТФ 83, 1,144 (2013).

[4] T. H. W. Goebel, T. W. Becker, D. Schorlemmer, S. Stanchits, C. Sammis, E. Rybacki, G. Dresen. J. Geophys. Res. 117, B03310 (2012).

[5] Xinglin Lei, Shengli Ma. Earthquake Science 27, 627 (2014).

[6] F. Chayes, Am. J. Sci. 248, 378 (1950)

[7] R. M. Stesky. Can. J. Earth. Sci. 15, 361 (1978).

[8] P. Churcher, P. French, J. Shaw. SPE International Symposium on Oileld Chemistry. SPE21044 (1991).

[9] Е.Е. Дамаскинская, В.Л. Гиляров, И.А. Пантелеев, Д.Р. Гафурова, Д.И. Фролов. ФТТ 60, 9, 1775 (2018).

[10] В.П. Ефимов. Физико-технические проблемы разработки полезных ископаемых 3, 11 (2006).

Редактор Т.Н. Василевская 